\title{
OCORRENCIA DE TRIPALEA CLAVARIA (Studer, 1878) NA PLATAFORMA CONTINENTAL \\ DO RIO GRANDE DO SUL
}

(Recebido em 28/12/1970)

LUIZ ROBERTO TOMMASI *

Instituto Oceanográfico da Universidade de São Paulo

SYNOPSIS

The occurrence and distribution of Tripalea clavaria (Studer, 1878)

is discussed, in relation to Brazilian and Falklands currents convergence and water mass in the South-western Atlantic.

IN TRODUÇÃO

O gênero Tripalea Bayer, 1955 (Anthozoa, Gorgonacea) foi erigido para abrigar a espécie Suberia clavaria Studer, 1878 é monotípico.

Caracteriza-se pelas colônias clavadas, sem ramificações, de côr branca. A cortex a presenta uma região externa delgada e uma in terna compacta, vesicular e com longas espículas. Os bastões medulares são furcados em amb as extremidades. As lacunas intercomunica doras são profundas.

A espécie era conhecida de $35^{\circ} 01^{\prime} \mathrm{s}$ $54^{\circ} 24,9^{\prime} \mathrm{W} ; 36^{\circ} 48^{\prime} \mathrm{S}-53^{\circ} 35^{\prime} \mathrm{W}$ (Studer,1878); Punta Medanos, Patagônia; Montevideo (Kükentha1,1919), Rio de Janeiro e ao largo de Mon tevideo (Bayer, 1961) e em várias posições entre $37^{\circ} 40^{\prime} \mathrm{s}-37^{\circ} 20^{\prime} \mathrm{s}$ e $56^{\circ} 30^{\prime} \mathrm{W}-58^{\circ} \mathrm{W}(01 \underline{i}$ vier et al., 1968, como Suberia sp.).

No presente trabalho, apresentamos algu mas observações sôbre a ocorrência desta es pécie na região sul do Brasil e discutimos seu limite norte de distribuição.

Pub1. no. 307 do Inst. Ocean. da USP.
OCORRENCIA

Nos trabalhos de prospecção pesqueira, realizados durante as viagens do N/Oc."Prof. W. Besnard" na plataforma continental do Rio Grande do Sul, dentro do convênio com o Grupo Executivo do Desenvolvimento da Indústria da Pesca (GEDIP), Rio Grande do Sul, foram e fetuados 162 arrastos com arrastão de portas. Em seis dêsses arrastos (Fig. 1) foi coletado o gorgonacea Tripalea clavaria (Studer, 1878) (Fig. 2). A Tabela seguinte apresenta os dados dos locais onde essa espécie foi en contrada.

A Tabela e a Figura 1 mostram que esta espécie ocorre até a altura de Sarita, não tendo sido encontrada ao norte dessa região, apesar de terem sido efetuados arrastos em tôda a área entre $29^{\circ} \mathrm{S}$ e $35^{\circ} \mathrm{S}$.

\section{DISTRIBUIÇ̃̃o}

Ocorreu em temperatura de 12,87 a $18,40^{\circ} \mathrm{C}$, salinidade de 32,83 a $35,97 \%$ e o teor de oxigênio de 4,48 a $5,9 \mathrm{~m} 1 / 1$ (ägua de fun do). Profundidade de 44 a 190 metros. Ocor-

* Bolsista do Conselho Nacional de Pesquisas, Pr. $9386 / 68$.

Bolm Inst. oceanogr. S Paulo, 20:27-32,1971 
reu sempre sôbre pedras ou valvas de lameli brânquios.

0 maior número de exemplares (Est.417) foi encontrado a $50 \mathrm{~m}$ de profundidade, tem peratura de $16,66^{\circ} \mathrm{C}$, salinidade de $34,78^{\circ} / 00$ e teor de oxigênio de $5 \mathrm{ml} / 1$ (ägua de fundo), mas na estação 460 , onde encontramos o segundo número de exemplares, a temperatura foi de $18,40^{\circ} \mathrm{C}$.

TABELA

\begin{tabular}{|c|c|c|c|c|c|}
\hline $\begin{array}{c}\text { Est ação } \\
(\mathrm{n} \stackrel{)}{ })\end{array}$ & $\begin{array}{c}\text { Exemp } 1 \text { ares } \\
(\mathrm{n} 9)\end{array}$ & $\begin{array}{c}\text { Profundidade } \\
\text { (m) }\end{array}$ & $\begin{array}{c}\text { Temperatura } \\
\left({ }^{\circ} \mathrm{C}\right)\end{array}$ & $\begin{array}{c}\text { Salinidade } \\
(0 / 00)\end{array}$ & $\begin{array}{c}\text { oxigênio } \\
(\mathrm{m} 1 / 1)\end{array}$ \\
\hline 320 & 5 & 47 & 12,99 & 33,07 & 5,34 \\
\hline 328 & 3 & 44 & 12,87 & 32,83 & 5,9 \\
\hline 330 & 1 & 148 & 17,70 & 35,97 & 5,0 \\
\hline 417 & 31 & 50 & 16,66 & 34,78 & 5,0 \\
\hline 419 & 1 & 190 & 15,67 & 35,73 & 4,7 \\
\hline 460 & 11 & 46 & 18,40 & 35,08 & 4,48 \\
\hline
\end{tabular}

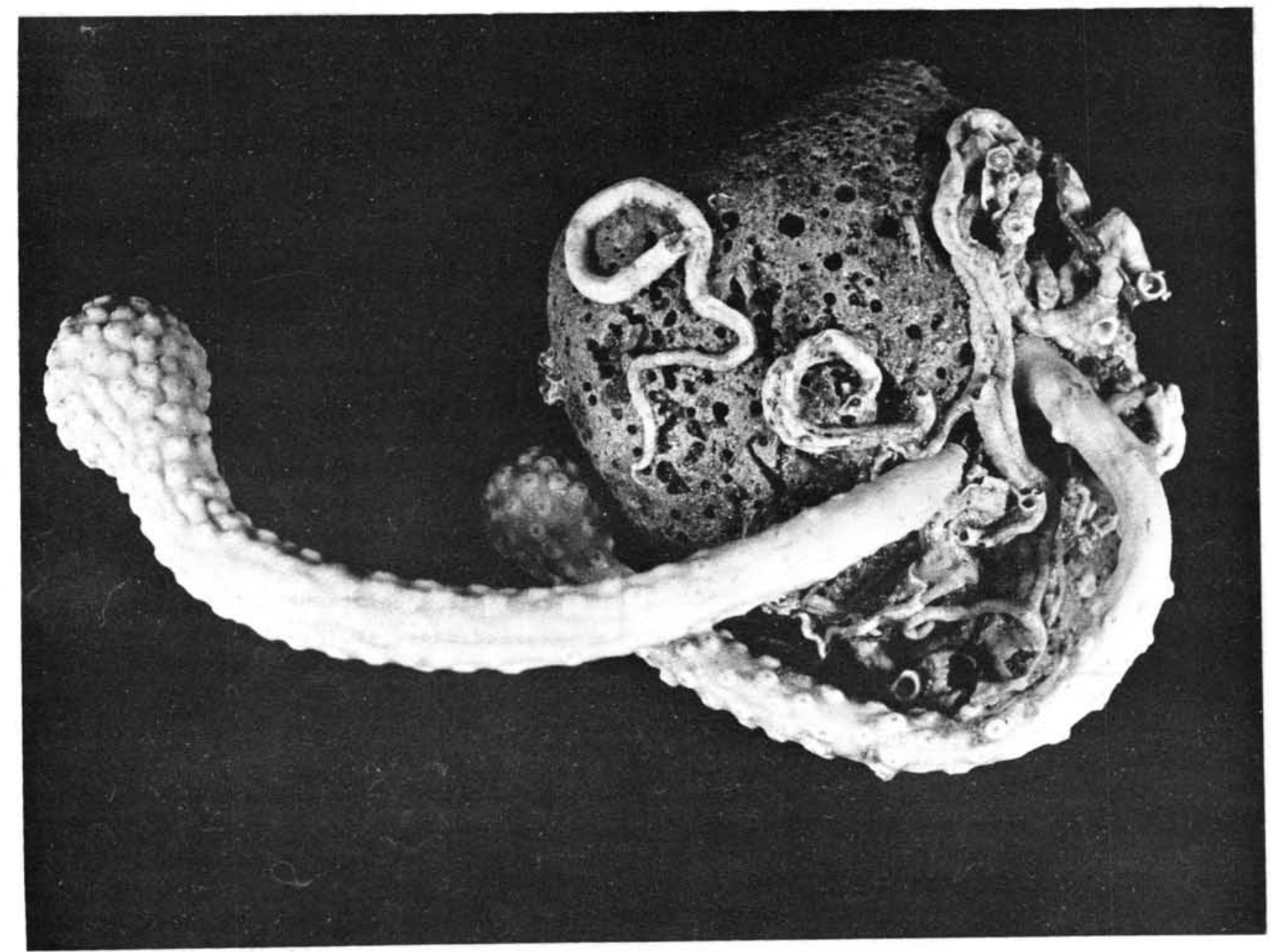

FIG. 2 - Vista geral de Tripalea clavaria. 
Foi por nós mostrado, em trabalho ante rior (Tommasi, no pre10) que a região de Mos tardas ou seja, aque 1 a aparentemente mais sujeita à influência do limite oeste da con vergência subtropical, se constitui em uma barreira à dispersão de elementos da fauna

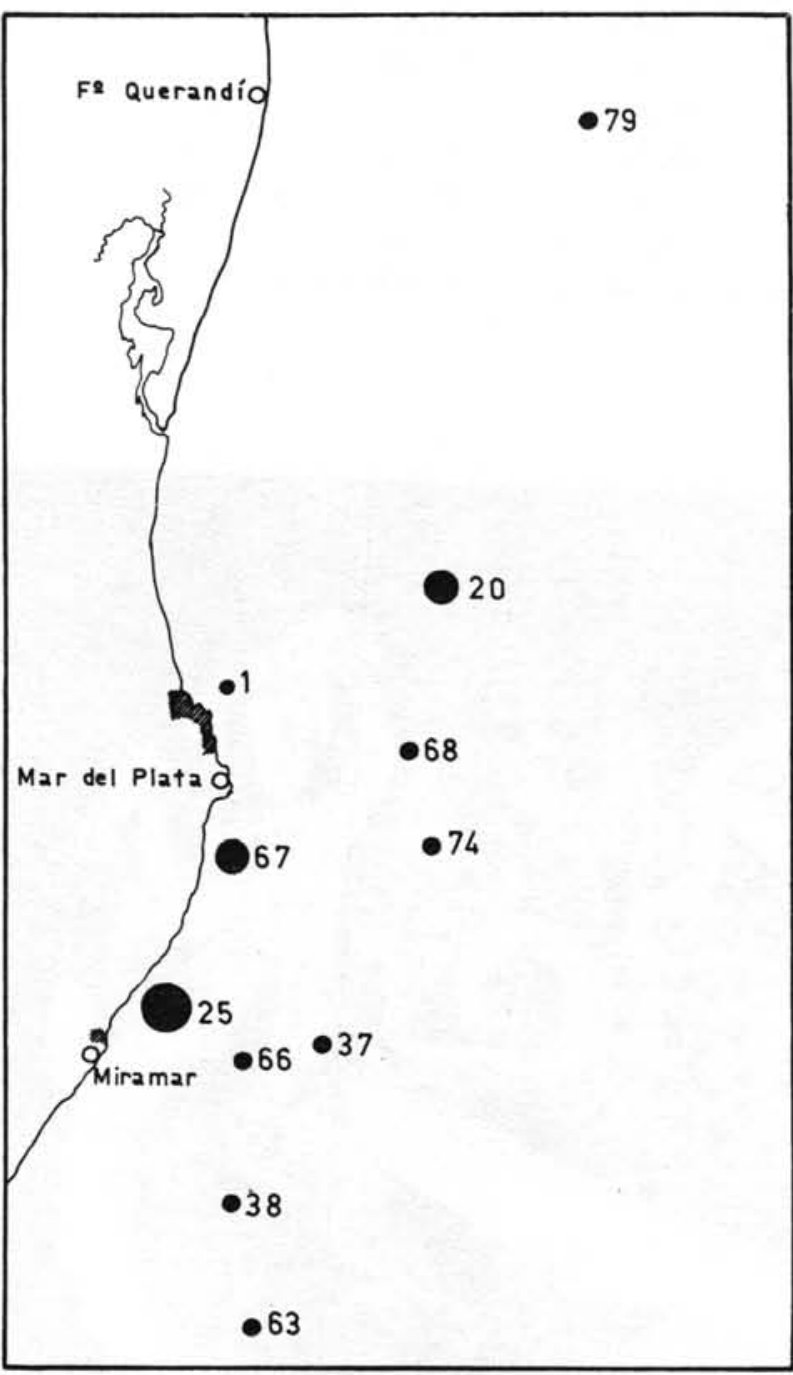

FIG. 3 - Posição das estações ao largo da Argentina, nas quais ocorreram Tripalea clavaria (segundo 01ivier et al., 1968). 0 diâ metro dos círculos representa a abundància da espécie nas diversas estaçöes.

tropical para o sul. A distribuição da Tripalea clavaria sugere que essa região seja também uma barreira à dispersão para o nor te, dessa espécie.

Segundo Olivier et al. (1968) foi cole tado na Argentina em 11 estações (Fig.3) en tre $37^{\circ} 30^{\prime} \mathrm{s}$ e $38^{\circ} 40^{\prime} \mathrm{s}$ (como Suberia sp.), sô bre rochas, conchas e organismos vivos. E característica da associação de Mytilus pla tensis-Lithophaga patagônica, de fundos du ros, ainda que ocorra acidentalmente em ou tras (0livier et al., op. cit.). Foi obtida nessa região de 18 a $35 \mathrm{~m}$ de profundidade, temperatura de 10,49 a $20,1^{\circ} \mathrm{C}$ (superfície), salinidade de 32,83 a $33,96^{\circ} / 00$ (superfície) e teor de oxigênio, de 6,52 a $6,69 \mathrm{ml} / 1$ (água de fundo). Ainda que a maioria dos dados de olivier et al., (op. cit.) se refiram à ägua de superfície e não de fundo, pode-se verificar, como era de se esperar, que ocorreu na região de Mar del Plata,em temperatura e salinidade mais baixa do que no Rio Grande do Sul.

Quanto à profundidade, ocorreu na Argen tina em regiões menos profundas, ou seja a té 18 metros. A não ocorrência em profundidades menores do que 40 metros, na região do Rio Grande do Sul, e os dados anteriormente citados, sugerem seu deslocamento para profundidades maiores, devido a presença, em profundidades menores, de àgua mais quente oriunda da corrente do Brasil e em profundi dades maiores, de águas mais frias, da contracorrente subtropical.

Sendo uma espécies criöfila, acorreria apenas em regiōes sob influência ou de àgua subantártica ou das Malvinas.

Para Bayer (1961) o limite norte de sua área de distribuição é o Rio de Janeiro ou seja, se extenderia para o norte até onde ocorra água subtropical. Os dados sugerem porém, que ao norte de Mostardas, sua ocorrência seja esporádica, em profundidades mai ores do que 50 metros e apenas em regiões co mo a do Rio de Janeiro, citada por Bayer, onde essa água ressurge e a da Ilha Vitória, onde a coletamos em 1968 a 50 metros de profundidade, em região também sob influência dessa āgua (ver Emilsson, 1959).

\section{GORGONACEA DO BRASIL}

Além da presente espécie, são conhecidas 13 espécies ( 7 gêneros) de gorgonaceas do Brasil. Em face da escassez de literatura especializada sôbre êsses antozoārios, cre mos ser interessante apresentar a seguir u ma chave para a identificação daqueles gêne ros (baseada em Kükentha1,1919 e em Bayer, 1961). As gorgonaceas conhecidas do Brasil, 
pertencem a três famílias, Plexauridae Gray, 1859 , Gorgoniidae Lamouroux, 1812 e Antho the 1 idae Broch, 1916.

1 - Axis com a região central dividida em

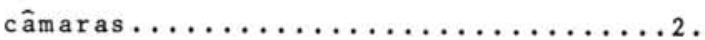

- Axis sem tais câmaras......... Antho thelidae (gênero Tripalea Bayer, 1955).

2 - As maiores espículas são bem desenvolvi das, geralmente bem maiores do que $0,2 \mathrm{~mm}$ de comprimento. A cortex do eixo é locu

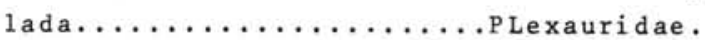

- As maiores espículas raramente atingem comprimentos superiores a $0,2 \mathrm{~mm}$. A cor tex do eixo ou não é loculada, ou é pou co loculada................ Gorgoniidae.

\section{P LE XAUR I DAE}

1 - As espículas predominantes da cortex são

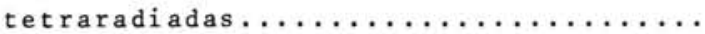
........Plexaure Zza Lamouroux, 1812 .

- As espécies predominantes da cortes,não

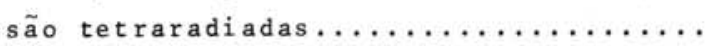
.......Muriceopsis Aurivil1ius, 1931.

\section{GORGONIIDAE}

1 - Com escafóides (espículas curvas,em for ma de canoa) presentes (Fig. 4)......

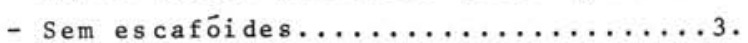
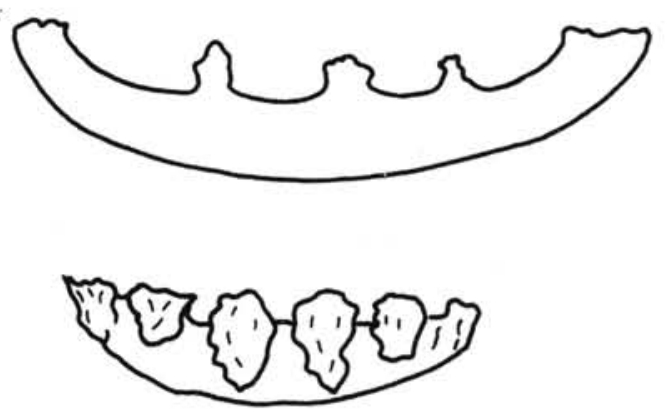

FIG. 4 - Espículas do tipo escafóide.

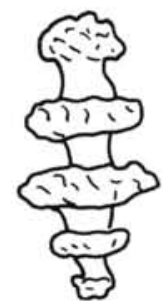

FIG. 5 - Espícūlas do tipo tubérculo fundido em discos.
2 - Ramificações do eixo, livres.......... ......Pseudopterogorgia Kükentha1,1919.

- Ramificações do eixo, anastomosadas.... $\ldots \ldots \ldots \ldots \ldots \ldots \ldots \ldots \ldots \ldots \ldots \ldots \ldots \ldots \ldots$

Zogorgia Milne Edwards \& Haime, 1850 .

3 - Com espículas corticais do tipo tubércu$10 s$ fundidos em discos (Fig. 5) ........ Leptogorgia Milne Edwards \& Haime, 1857.

- Sem tais espículas ..............

4 - Ramificações do eixo, 1ivres.......... Lophogorgia Milne Edwards \& Haime, 1857.

- Ramificações do eixo, anastomosadas.... ............Pacifigorgia Bayer, 1951.

S UMMARY

The region of the convergence between the Brazilian and the Falklands currents is a barrier to the northern distribution of Tripalea clavaria (Studer, 1878). It occurs in the Rio Grande do Sul plataform, in deepest regions that in Argentina plataform.This probably occurs, because of highest temperature of shallow waters of Rio Grande do Sul.

A key to the gorgonacea families and genera known from Brazil is presented.

\section{B I BL I OGRAF IA}

BAYER, F.M.

1961. The shallow-water Octocorallia of the West Indian region. The Hague, Nijhoff, $373 p$.

EMILSSON, I.

1959. Alguns aspectos físicos e químicos das águas marinhas brasileiras. Ciênc. Cult., S Paulo, vol. $11, \mathrm{n}$ ㅇ. $2, \mathrm{p} .44-54$.

KÜKENTHAL, $W$.

1919. Gorgonaria. Wiss. Ergebn. dt. Tiefsee - Exped. "Valvidia", vol. $13, \mathrm{n}$ ? $2,646 \mathrm{p}$.

OLIVIER, S.R., BASTIDA, R. \& TORTI, M.R.

1968. Resultados de 1 as campañas oceanograficas Mar de 1 Plata I-V.Con tribución al trazado de una carta bionómica de 1 área de Mar del P1ata. Las asociaciones de 1 sistema 1 itoral entre 12 y $70 \mathrm{~m}$ de profundidad. Boln Inst. Biol. mar., no $16,85 \mathrm{p}$.

STUDER, T.

1878. Uebersicht der Anthozoa Alcyonaria, welche während der Reise SMS Gazelle um die Erde gesam- 
melt wurden. Mber. K. Preuss.

Akad. Wiss. Berl., p.632-688,

5 est.

TOMMASI, L.R.

Filtros ecológicos e barreiras biogeográficas à dispersão da fauna bêntica marinha no At lân tico Sul 0cidental. Inst. oceanogr. USP (no prelo) 